\section{Stem Cells in Biology and Disease ESTOOLS International Symposium}

\author{
Lisbon, 26-28 May 2010
}

ESTOOLS was the first EU-funded project entirely devoted to the study of human embryonic stem cells (ESCs) and induced pluripotent stem cells (iPSCs). The final meeting of the consortium took place in Lisbon from 26 to 28 May 2010 and gathered 298 researchers from 27 countries. A dense programme of scientific sessions and outreach activities, together with an ethics workshop gave an all-round overview of the challenges, perspectives and ethical issues of this exciting field.

\section{Introduction}

The mournful lyrics of fado, the traditional music resonating from the old bars of the Alfama district in Lisbon, remind listeners that destiny is immutable - fado itself is the Portuguese word for "fate". Stem cell researchers attending the ESTOOLS symposium last May in Lisbon would disagree with such a deterministic attitude. Indeed, most of their work is aimed to control fate, at least that of human cells, either by directing the differentiation of embryonic stem cells (ESCs) into the desired types of adult tissue, or, vice-versa, by reprogramming adult cells back into a pluripotent state.

The ESTOOLS consortium was launched in 2006 as a 4-year EU-funded 6th Framework (FP6) project, with a total funding of 12 million euro. The project has been the largest European consortium devoted to the study of human ESCs, teaming up 21 research groups in 10 countries. After the discovery of human induced pluripotent stem cells (iPSCs) by Shinya Yamanaka and James Thomson in 2007, the consortium opened a new workpackage dedicated to this groundbreaking field, and became the first to devote EU funding to study human iPSCs. The Lisbon meeting marked the end of the consortium's funding cycle, but it was more than a wrap-up of its overall work and achievements, providing a showcase of cutting-edge research in human pluripotent stem cells, with the participation of international leaders in the field.

Human pluripotent stem cells (ESCs and iPSCs) offer unprecedented opportunities for regenerative medicine and the bioindustry, because of their capacity to self-renew and turn into virtually any type of mature cell. While the therapeutic potential of human ESCs is clear, the first and only (so far) clinical trial involving a hESCs-derived drug (GRNOPC-1 from Geron Corp.) was approved by the FDA in 2009 and enrolled its first patent in October 2010. In order to advance towards clinical applications, more basic research is needed to address the mechanisms of

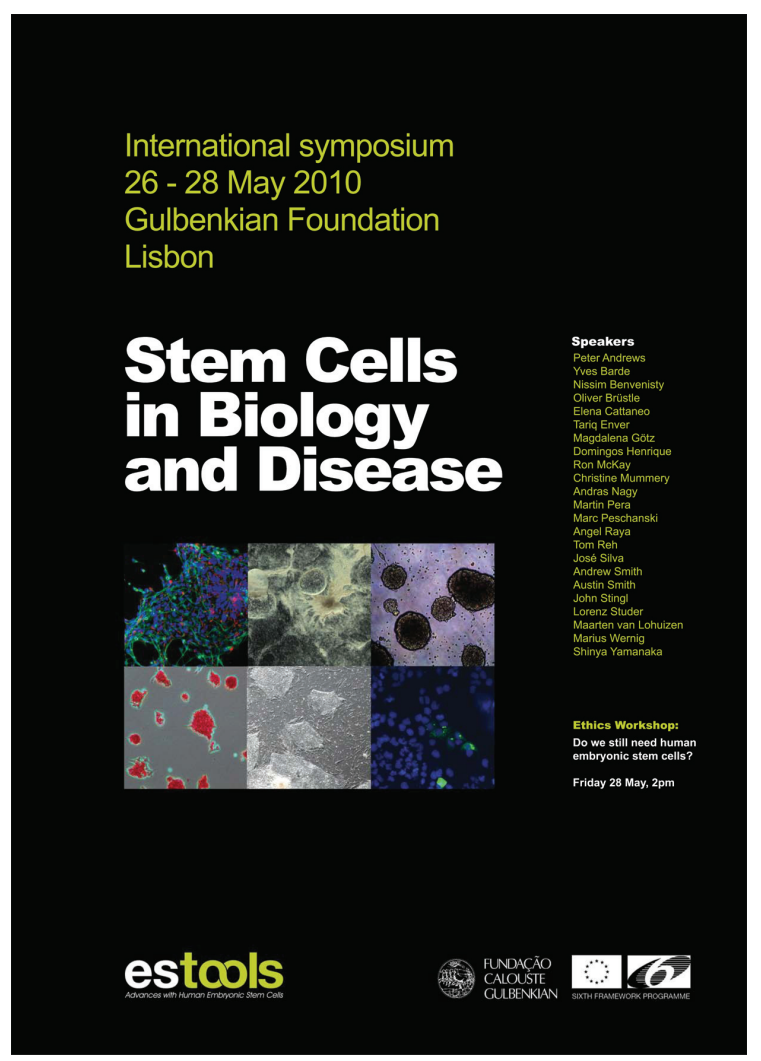

self-renewal, commitment and differentiation of human pluripotent stem cells. As its acronym suggests the mission of ESTOOLS was to provide a range of cognitive and practical tools in such a direction; its laboratories effectively contributed to advancing knowledge of human pluripotent stem cells and developed a range of much sought-after protocols to grow, modify and differentiate human ESCs.

Outside research laboratories, human ESCs are equally renowned for their potential to heal and for the ethical and political controversy regarding their use. With this in mind, the symposium hosted an Ethics Workshop (ethics research was part of the consortium's core activities) and a series of outreach initiatives bringing stem cells science to the larger public, including the premiere of the theatre play Staminalia (see below). The fascinating Fundação Calouste Gulbenkian, a landmark of modern Lisbon, where conference rooms are at a doorstep to paintings and sculptures of the adjacent Gulbenkian Museum, hosted the sym-

Abbreviations used in this paper: CSC, cancer stem cell; ESC, embryonic stem cell; iPSC, induced pluripotent stem cell.

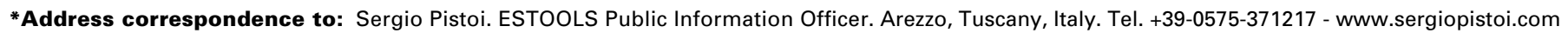
e-mail: sergio@sergiopistoi.com
} 
posium: such an environment greatly contributed to creating an original and fertile interaction between Science and Art.

\section{Be fruitful and multiply}

A significant part of ESTOOLS' work was devoted to elucidate the mechanisms of human ESCs' self renewal and to develop new and more effective protocols for expanding these cells while retaining their pluripotent state.

ESCs grown in vitro are subject to the so-called culture adaptation, a phenomenon by which some genetic variants that favor self-renewal are selected after multiple passages, leading to genetic abnormalities that need to be carefully monitored and investigated. Studying culture adaptation may also provide insights into the mechanisms controlling self-renewal and differentiation of ESCs. Peter Andrews (University of Sheffield and ESTOOLS' coordinator), reported a collaborative study through the ESTOOLS consortium that mapped hundreds of copy number variations (CNV) and loss of heterozygosity ( $\mathrm{LOH}$ ) in 17 human ESCs lines, and provided a shortlist of genes mapping inside or near the mutated sites (Narva et al., 2010). Some of the genetic abnormalities seen in human ESCs are also often observed in embryonal carcinoma cells, the stem cells responsible for germ cell tumors. The work, said Andrews, will help to shed light on the adaptation process of human ESCs in culture and will make it easier to choose ESC in which crucial genes are less likely to mutate.

Another issue is posed by the so-called "feeder" cells, mouse or human fibroblasts which are co-cultured with ESCs, providing them with growth factors and with an attachment substrate. Feeder cells, and serum, which is also added to ESCs cultures, may contaminate cultures with pathogens, hampering their use in patients. A remarkable contribution from ESTOOLS consortium was the identification of recombinant laminin-511 as an effective attachment substrate for growing human ESCs: combined with the use of serum substitutes, like heparin, this allowed feeder- and serum-free expansion of human ESCs (Vuoristo et al., 2009),(Furue et al., 2008).

The molecular switches governing self-renewal and differentiation of human ESCs were the topic of a report by Oliver Brüstle (University of Bonn and ESTOOLS partner). His group recently showed that turning off Notch-mediated signals promotes neural differentiation of human ESCs (Borghese et al., 2010). Together with other results, also obtained by the consortium, this work identifies Notch as a key regulator of human ESCs self-renewal and neural differentiation.

\section{From human ESCs to neurons and beyond}

Directing the fate of human ESCs into different neural populations was one key goal of ESTOOLS, and the meeting provided a riveting gallery of recent technological advances in the field, showing an array of recent protocols (some developed thorough the consortium), that make feasible to obtain a variety of neuronal cell types from human ESCs.

Moving a step further, Lorenz Studer (Memorial Sloan-Kettering Cancer Center, New York) reported how he was able not only to obtain human ESC-derived neural precursors, but also recreated complex, functioning developmental organizers in vitro. His group used two inhibitors of SMAD signaling, Noggin and SB431542, to induce rapid and efficient (>80\%) neural conversion of human ESCs. Neural precursors thus obtained were then successfully used to reproduce the floor plate, a key signaling center for neural development which is normally located on the ventral midline of the embryonic neural tube. The ESC-derived floor-plate is the first example of an in vitro-generated human developmental organizer and displays markers and regional identity similar to that of its natural counterpart (Fasano et al., 2010). Using the same in vitro platform, Studer's group also recreated the anterior ectodermal placode tissue, an embryonic structure that gives rise to important neuronal cells of the head, including olfactory neurons, hormoneproducing anterior pituitary cells, lens cells and cranial nerve neurons.

\section{Tracking cancer stem cells}

The existence of cancer stem cells (CSCs) is an old postulate in tumor biology which was recently revived due to the availability of new molecular markers. According to the CSC model tumors contain a subpopulation of self-renewing malignant cells which is responsible for starting and maintaining cancer growth. Such a model is in contrast to the more classical idea that tumor cells are equivalent, and become tumorigenic following stochastic "hits" (mutations, external factors etc.). However, the existence of CSCs may explain why many tumor therapies fail: drugs targeting the bulk of a tumor may temporarily destroy most cancer cells, but the self-renewing subpopulation, if left intact, would lead to subsequent relapse (Dick, 2009).

Tariq Enver (University of Oxford and ESTOOLS partner) and John Stingl (Cancer Research UK, Cambridge) provided revealing examples of how the CSCs model could be applied to childhood acute leukemia and mammary tumors, respectively. Acute lymphoblastic leukemia (ALL) is the most common childhood tumor in developed countries, and is currently treated with chemotherapy. In most patients affected by ALL, leukemic cells contain a chromosomal translocation leading to the fusion of TEL and AML1 genes. There is also evidence that the TEL-AML1 is the initiating event for leukemogenesis in utero, giving rise to preleukemic clones that are subsequently expanded by poorly-known factors (infections being a likely candidate). Enver's group found that ALL patients possessed a rare pre-leukemic cells which expressed markers typical of both hemopoietic stem cells and the B-cell lineage, calling them STEM B cells. The two STEM B markers allowed researchers to track this subpopulation in different situations. Two years ago, the group found a pair of twins of which only one had developed leukemia; both siblings, however, carried STEM B clones that were passed in utero through the common placenta: the healthy twin therefore provided the first example of pre-leukemic child (Hong et al., 2008). Researchers also obtained in vitro STEM B models using cord blood stem cells transfected with the TEL-AML1 fusion gene, and were able to induce the expansion of these pre-leukemic clones with TGF-beta, one of many factors secreted upon infection. Interestingly, both in vitro and in vivo studies showed a very high genetic heterogeneity of STEM B cells, leading Enver to caution that we should not be too dogmatic about CSCs, as they could vary across disease types and even within the same particular tumor, making them particularly elusive. Are STEM B cells responsible for relapse after treatment of ALL, anyway? To answer this question, 


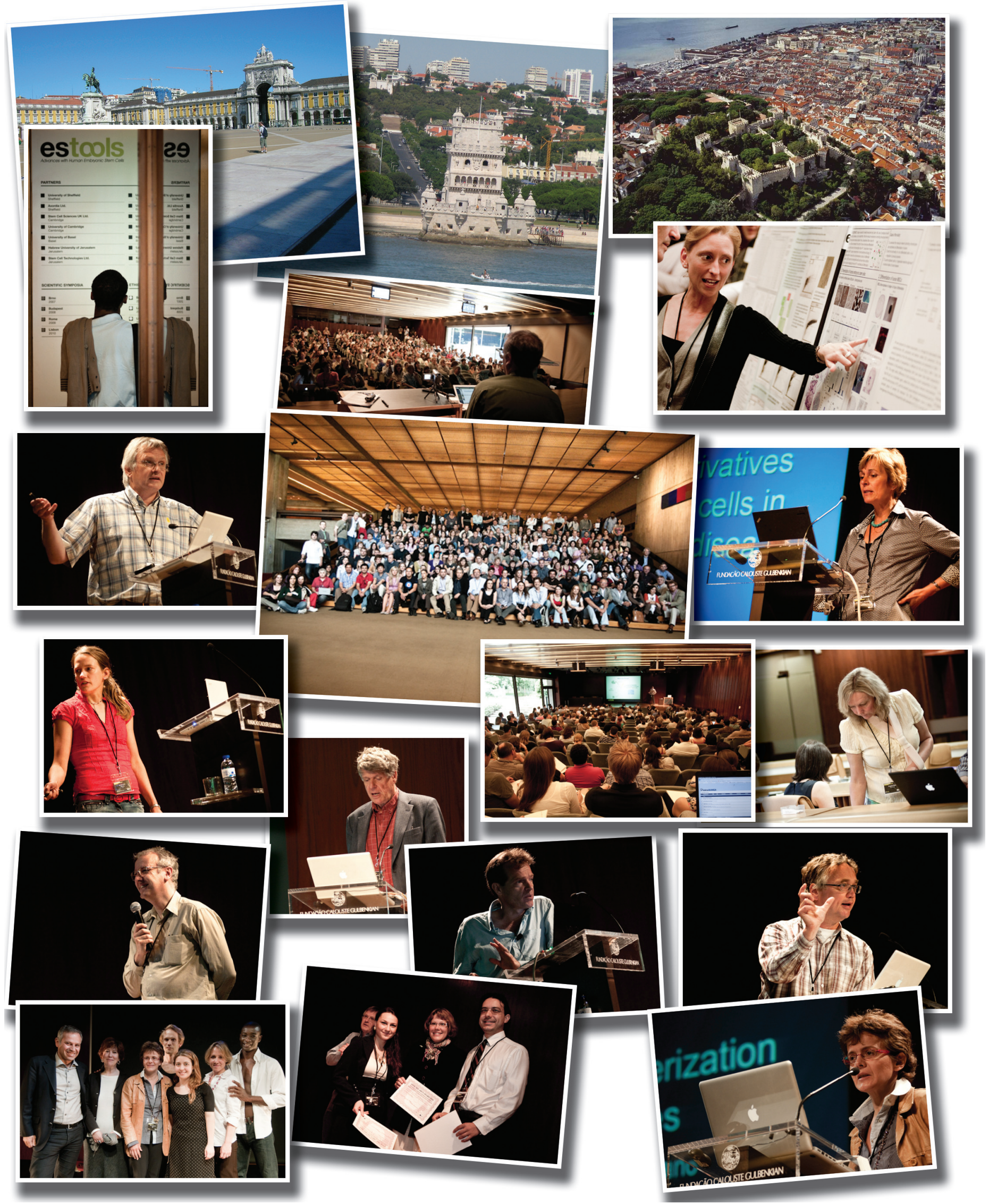


Enver's group will collect samples at various stages, from diagnosis to the end of treatment, from several patients in the next 8 years. Early data showed that subpopulations of STEM B cells are resistant to chemotherapy and are found in relapsing patients.

John Stingl reported advances in the search and characterization of CSCs from breast tumors. A cancer stem cell component has already been found in these tumors; despite the genetic heterogeneity of CSCs, Stingl hopes to find specific stem cell "signatures" that correlate with different types of tumors of different levels of malignancy. This, said Stingl, could ameliorate prognostic and help with choosing the most effective therapy for breast tumors patients.

\section{Pluristratified exploits}

If you think which organ is easier to obtain from stem cells, the skin would probably be the first that comes to mind. Cell therapy with epidermal substitutes for treating severe burns has been a reality for more than 25 years and, today, large surfaces of epidermis are routinely obtained by culturing a few $\mathrm{cm}^{2}$ of the patients' skin for autologous transplant. Despite these encouraging bases, many years of unsuccessful attempts were needed before the first human ESCs-derived epidermis was obtained in 2009.

Marc Peschanski (I-STEM, INSERM, Paris), whose group created the first human pluristratified epidermis from ESC-derived keratinocytes (Guenou et al., 2009) discussed these recent results and other advances in the field. The histological and time-frame expression of several developmental markers in the ESC-derived epidermis was identical to that of wild type human skin, said Peschanski. He stressed that respecting chronobiology was key to their success: ESCs were induced for 40 days, the time in which the epidermis normally forms in embryos. Interestingly, the French team could also selectively induce differentiation of human ESCs into melanocytes, that produced and transferred melanosomes into the newly-obtained epidermis, providing it with pigmentation. Peschanski said that his group is now performing preclinical studies on macaque models, testing tolerance of allogenic grafts of ESC-derived epidermis. He admitted, however, that there is no consensus yet about the opportunity of using ESCs-derived epidermis in patients: some clinicians believe that allogenic skin grafts would be more risky, and not more beneficial, than the autologous transplant that are currently in use (Pellegrini and De Luca, 2010). Peschanski, instead, pointed to the advantages of ESCs-derived grafts, arguing that growing a sufficient amount of autologous skin can currently take many days, during which the burn patients are left vulnerable to infections; moreover, he said, current cell therapies are neither directly applicable to inherited skin diseases, such as epidermolysis bullosa, nor they are suitable for industrialization. Given the difficulty of predicting technological outcomes in the long run, only time will probably tell in which applications, if any, ESCderived grafts will become the new golden standard.

\section{Pushing the envelope on iPS cells}

Shinya Yamanaka (Kyoto University) showed in 2006 that adult mice fibroblasts can be genetically reprogrammed with only four factors (Oct3/4, SOX2, KIf4 and c-Myc) to become pluripotent cells, opening the groundbreaking field of induced pluripotent stem cells
(iPSCs). One year later, his group and that of James Thomson in Wisconsin independently obtained iPSCs from human fibroblasts. These cells pass high stringency tests for pluripotency, including germline transmission and tetraploid complementation, and share many features with ESCs.

Yamanaka delivered his keynote speech from Japan through a web video link (the disruption of his travel plans was the only notable consequence of the Eyjafjallajökull's ash on the Lisbon meeting) and reviewed the therapeutic perspectives of using iPSCs. Preclinical studies with human iPSCs recently provided encouraging proofs of principle for the treatment of sickle cell anemia, Parkinson's disease and spinal chord injuries in animal models (Hanna et al., 2007; Tsuji et al., 2010). Angel Raya (Centre for Regenerative Medicine, Barcelona) reported in a separate talk that his group corrected the genetic defect causing Fanconi anaemia in the somatic cells taken from patients and then reprogrammed them into patient-specific iPS cells that were indistinguishable from that of healthy individuals. These cells gave rise to normal myeloid and erythroid progenitors with potential value for the cell therapy of the disease (Raya et al., 2009).

Besides these promising results, several talks pointed out to the bottlenecks that need to be addressed before iPSC-based therapies will be transferred to patients. Strategies for iPSCs induction need to be improved (only about $0,05-0,08 \%$ of fibroblasts are currently transformed into iPSCs) and so do methods to prospectively purify different iPSC-derived cell types. Safety issues are posed by retroviral vectors, which are used to deliver inducing factors, as it is known that rogue integration of these vectors may be carcinogenic. Alternative methods for obtaining iPSCs, which employ adenoviral or non-viral delivery systems, have been recently developed to rule out such danger (Kaji et al., 2009; Woltjen et al., 2009).

Unlike ESCs, iPSCs offer the perspective of tailor-made cell treatments: pluripotent stem cells can be generated from the fibroblasts of an individual, and then transplanted back to the same person, avoiding immune rejection. Personalized iPSCs therapies are theoretically possible, but may be too expensive and timeconsuming to be practically applied on patients, said Yamanaka. As an alternative, he proposed iPS cells banks, i.e. large collections of ready-to-use iPS cells lines obtained from donors with different HLA haplotypes, that would ensure compatibility with most patients. The Kyoto iPS Cell Bank, which Yamanaka is setting up at his University, will contain iPSCs from 50 unique HLA homozygote donors; Yamanaka estimates that, in this way, the bank's samples will be compatible with about $90 \%$ of the Japanese population.

While clinical applications are still on the drawing board, disease modeling is the field where iPSC have already proven useful: iPSC lines generated from patients with a variety of disorders are today invaluable tools for performing studies that would be otherwise difficult or impossible to do in humans. ESTOOLS partners Elena Cattaneo (University of Milan) and Nissim Benvenisty (University of Jerusalem) contributed to this burgeoning field by generating several iPSC lines from Huntigton's Disease and Fragile $X$ patients, respectively. Lorenz Studer reported to have used homologous recombination to knock out specific genes in iPSCs; this technique, he said, will make possible to introduce the same mutation in lines with different genetic backgrounds, studying the effects of complex gene interactions. 
The symposium also tried to address a major question: do iPSCs from older donors differ in their biological of functional characteristics? Results reported by Yamanaka suggest that the donor's age does not affect the efficiency by which iPSCs are obtained. However, these data regarded a limited number of donors and all experts agreed that it still too early to draw firm conclusions. What is the effect of aging in iPSCs cultures? What is the survival/engrafting potential of human iPSCs compared to ESCs? Future studies will hopefully answer these and other key questions.

\section{Transdifferentiation: the new kid on the block?}

Somatic cells can be brought back to their pluripotent state and then, induced to differentiate into new adult cells, as showed by the advent of iPSCs. Transdifferentiation is a poorly understood phenomenon in which adult cells switch directly from a tissue type into another, skipping the pluripotent state altogether. Instances of transdifferentiation are observed naturally in vertebrates and occur in humans, for example in Barrett's metaplasia, in which the normal squamous epithelium of the esophagus is replaced by metaplastic columnar epithelium. In vitro examples include the direct conversion of fibroblasts into myocytes induced by transcription factor MyoD, known since the late 1980s, and, more recently, the direct switch of fibroblasts to macrophages and that of lymphoid to myeloid progenitors.

Why not harness transdifferentiation to obtain a quick fateswitcher for somatic cells, then? Marius Wernig (Stanford University, USA) reported how his group used a combination of five factors to induce direct transdifferentiation of human fibroblasts into neuronal cells that passed golden standard tests for pre- and post-synaptic neuronal function, like their natural counterparts.

Transdifferentiation, reminded Wernig, challenges our ideas about the epigenetic mechanisms underlying cell fate and reprogramming. The classical idea of development, was illustrated in the 1950s by Conrad Waddington's metaphoric "epigenetic landscape": in this model, developing cells were represented as marbles rolling down from a mountain with many valleys, each representing a different developmental path that the cells could take while moving downhill towards differentiation. Until recently, it was assumed that reprogramming worked mainly by erasing the epigenetic marks from differentiated cells, turning them back to their totipotent state, the equivalent of moving the marbles back to the top of Waddington's landscape. Transdifferentiation suggests a different picture, where cells jump directly from an epigenetic valley to another, without taking any step back to a pluripotent stage: reprogramming would not work by erasing the cells' epigenetic state, but rather, would function as an "extreme makeover" by adding new epigenetic marks to the existing ones (Zhou and Melton, 2008).

Transdifferentiation is a further proof that there is still a lot to learn about the epigenetic mechanisms of differentiation and reprogramming. "Both iPSC induction and transdifferentiation", said Wernig "are still very slow and inefficient, indicating that we still don't know much about what's going on".

\section{Do we still need human ESCs?}

The above question was the core theme of the ESTOOLS
Ethics Workshop, an event which accompanied the scientific programme in Lisbon. After the advent of iPSCs many people, especially those who are against research on human embryos, would rush to give a negative answer. Opposers to human ESCs research were indeed among the first to jump on the iPS bandwagon: if iPSCs and ESCs are identical, they argue, why should we pursue research on embryo-derived cells? The answer, of course, should not be based on ideological or religious beliefs or hopes, but on sound scientific evidence. To this regard, the Workshop, organized by Göran Hermerén (Lund University and ESTOOLS' ethics director), reviewed the similarities and differences between human ESCs and iPSCs on the basis of recent scientific studies (Hermerén and colleague Kristina Hug also presented a working paper of the subject). While there are striking similarities between the two types of cells, a number of recent studies revealed important differences between human iPS and ES cells. A first-hand account came from Nissim Benvenisty (Hebrew University of Jerusalem and ESTOOLS partner): his group recently compared iPSCs and ESCs from patients and embryos affected by Fragile $X$ syndrome, the most common form of inherited mental retardation in boys, and discovered that iPSCs did not mimic the disease as faithfully as their embryonic counterparts. The FMR1 gene, which is mutated in Fragile $X$ syndrome, was found to be active in ESCs from affected embryos: this was expected, since the gene is normally silenced only upon differentiation. By contrast, the same gene remained silenced in iPSCs, indicating that, for reasons that are still unknown, FMR1 resisted epigenetic reprogramming during iPSC induction (Urbach et al., 2010). Marc Peschanski, too, reported that the efficiency by which he obtained human epidermis from iPSCs was about half than with ESCs, and other recent studies comparing human iPSCs and ESCs side-to-side confirmed notable differences between the two models (Vogel, 2010). It is not clear if such differences are intrinsic to iPSCs or are due to the reprogramming protocols that need to be improved. Summing up all the available evidence, however, the prevailing conclusion at the symposium was that human iPSCs are not ready to fully replace their embryonic counterparts yet.

Squabbles over the use of human ESCs will not end there, of course. Indeed, Giuseppe Testa (University of Milan and ESTOOLS Ethics advisor) anticipates that the current wrangling over the use of embryo-derived cells will be just a sample of the debates that will follow the progress of stem cell research. The advent of stem cell technologies, he said, conflated with skyrocketing developments in sequencing methods and this combination, for the first time, exposed our cells not only to investigational scrutiny, but also to genetic manipulation. That is why new developments, said Testa, will require a "reprogramming of our scientific and societal choices". A few words from poetess April Mahoney conveyed a very similar idea: "we must be like the stem cells we seek/extracted out, allowed to reproduce/heal, cure, and change", read a large print on a banner along the main corridor at Gulbenkian taken from one of her poems (Mahoney, 2009). Interestingly, visitors at the Gulbenkian could see how science, ethics and poetry sometimes converge.

\section{Engaging the public}

During its four years of activity, the ESTOOLS programme 
implemented a range of outreach initiatives to disseminate information and facilitate discussion about the opportunities and issues of human ESC research. The T.E.L.E.S.C.O.P.E. program involved high school classes across Europe and facilitated discussion among students in different countries by harnessing inexpensive web video links. Smile of a Stem Cells, an exhibition of captivating photographs of stem cells from ESTOOLS' laboratories, transformed scientists into visual artists and toured in several European cities, taking cutting-edge science to the public. Both events were also performed in Lisbon with the collaboration of the Calouste Gulbenkian Foundation and the Gulbenkian Institute of Science.

At the crossroad between science and art was a premiere of the theatre play Staminalia, A Dream and a Trial, written and directed by Italian playwright Valeria Patera, which unfolded at the main Gulbenkian auditorium. The story was inspired by the Italian book Staminalia, written by philosopher Armando Massarenti (Massarenti, 2008) and by Elena Cattaneo, an ESTOOLS partner and a tenacious advocate of human ESCs research. The protagonist of Staminalia (played by Italian American actress Cariddi Nardulli) is a stem cell researcher and a mother who sees ethical issues and political limitations impacting into her scientific freedom, and into her private life. Far from being didactic, Staminalia was a powerful representation of the inextricable link between science, philosophy, politics and personal dilemmas that characterize stem cell research. Drama was intertwined with a unconventional mix of music, video and ballet, in which two near-naked dancers incarnated the scientists' conflicting and alternating feelings: unapologetic stance vs. painful doubts. The dancer's vigorous presence on stage was also a reminder that, after all, our body is the starting point, and the finishing line, of biomedical research.

\section{Sergio Pistoi}

Biomedical writer and consultant. ESTOOLS Public Information Officer

\section{Note}

The ESTOOLS symposium organizers would like to thank all attendees for participation and valuable discussions, and all speakers for sharing their published and unpublished data. They grateful acknowledge the Instituto Gulbenkian de Ciência and the Fundação Calouste Gulbenkian for assistance in developing the event and for hosting the meetings, and The International Journal of Developmental Biology (Int. J. Dev. Biol.) for sponsoring the Poster Prizes. The author apologizes to the many speakers whose work could not be included in the report, due to space limitations and would particularly like to thank Peter W. Andrews and Andrew Smith for their critical review of this manuscript. Details on the ESTOOLS project are available at: www.estools.eu

\section{IJDB poster awards}

The International Journal of Developmental Biology sponsored three poster prizes which where awarded collectively by the Symposium Scientific Committee and the ESTOOLS Scientific Advisory Board. 1st prize: Elsa Abranches (Biologia do Desenvolvimento, Instituto de Medicina Molecular, Portugal)

2nd prize: Jorge M. Pacheco (Departamento de Matematica e Aplicacoes, Universidade do Minho, Portugal)

3rd prize: Yvonne Fischer (Laboratory Medicine, University of Lund, Sweden).

Winners were offered complimentary Int. J. Dev. Biol. subscriptions and publishing packages.

KEY WORDS: ES cell, iPSC, CSC, meeting report

\section{References}

BORGHESE, L.et al. (2010). Inhibition of notch signaling in human embryonic stem cell-derived neural stem cells delays G1/S phase transition and accelerates neuronal differentiation in vitro and in vivo. Stem Cells 28: 955-964.

DICK, J. E. (2009). Looking ahead in cancer stem cell research. Nat Biotechnol 27 44-46.

FASANO, C. A., S. M. CHAMBERS, G. LEE, M. J. TOMISHIMA, AND L. STUDER (2010). Efficient derivation of functional floor plate tissue from human embryonic stem cells. Cell Stem Cell 6: 336-347.

FURUE, M. K.et al. (2008). Heparin promotes the growth of human embryonic stem cells in a defined serum-free medium. Proc Natl Acad Sci USA 105: 13409 13414

GUENOU, H.et al. (2009). Human embryonic stem-cell derivatives for full reconstruction of the pluristratified epidermis: a preclinical study. Lancet 374: 17451753.

HANNA, J.et al. (2007). Treatment of sickle cell anemia mouse model with iPS cells generated from autologous skin. Science 318: 1920-1923.

HONG, D.et al. (2008). Initiating and cancer-propagating cells in TEL-AML1associated childhood leukemia. Science 319: 336-339.

KAJI, K., K. NORRBY, A. PACA, M. MILEIKOVSKY, P. MOHSENI, AND K. WOLTJEN (2009). Virus-free induction of pluripotency and subsequent excision of reprogramming factors. Nature 458: 771-775.

MAHONEY, A. (2009). Connected at the cellular level. In Utter Magic, A Good Person Publishing, San Diego.

MASSARENTI, A. (2008). Staminalia. Guanda, Milan.

NARVA, E.et al. (2010). High-resolution DNA analysis of human embryonic stem cell lines reveals culture-induced copy number changes and loss of heterozygosity. Nat Biotechnol 28: 371-377.

PELLEGRINI, G., AND M. DE LUCA (2010). Human embryonic stem cell-derived keratinocytes: how close to clinics? Cell Stem Cell 6: 8-9.

RAYA, A.et al. (2009). Disease-corrected haematopoietic progenitors from Fanconi anaemia induced pluripotent stem cells. Nature 460: 53-59.

TSUJI, O.et al. (2010). Therapeutic potential of appropriately evaluated safeinduced pluripotent stem cells for spinal cord injury. Proc Natl Acad Sci USA 107: 12704-12709.

URBACH, A., O. BAR-NUR, G. Q. DALEY, AND N. BENVENISTY (2010). Differential modeling of fragile $X$ syndrome by human embryonic stem cells and induced pluripotent stem cells. Cell Stem Cell 6: 407-411.

VOGEL, G. (2010). Stem cells. Reprogrammed cells come up short, for now Science 327: 1191.

VUORISTO, S.et al. (2009). Laminin isoforms in human embryonic stem cells: synthesis, receptor usage and growth support. J Cell Mol Med 13: 2622-2633.

WOLTJEN, K.et al. (2009). piggyBac transposition reprograms fibroblasts to induced pluripotent stem cells. Nature 458: 766-770.

ZHOU, Q., AND D. A. MELTON (2008). Extreme makeover: converting one cell into another. Cell Stem Cell 3: 382-388. 


\section{Further Related Reading, published previously in the Int. J. Dev. Biol.}

See our recent Special Issue Developmental Hematopoiesis

edited by Charles Durand, Tierry Jaffredo and Alexander Medvinsky at: http://www.ijdb.ehu.es/web/contents.php?vol=54\&issue=6-7

1st Joint Meeting of the French \& Spanish Developmental Biology Societies (2009)

Alain Vincent and Eric Agius

Int. J. Dev. Biol. (2010) 54: 555-559

Feeder- and serum-free establishment and expansion of human induced pluripotent stem cells Mehdi Totonchi, Adeleh Taei, Ali Seifinejad, Mohammadsharif Tabebordbar, Hassan Rassouli, Ali Farrokhi, Hamid Gourabi, Nasser Aghdami, Ghasem Hosseini-Salekdeh and Hossein Baharvand

Int. J. Dev. Biol. (2010) 54: 877-886

Neurogenic and mitotic effects of dehydroepiandrosteroneon neuronal-competent marrow mesenchymal stem cells Esmaeil H. Shiri, Narges-Zare Mehrjardi, Mahmood Tavallaei, Saeid K. Ashtiani and Hossein Baharvand Int. J. Dev. Biol. (2009) 53: 579-584

\section{Primordial germ cell biology at the beginning of the XXI Century}

Massimo De Felici

Int. J. Dev. Biol. (2009) 53: 891-894

1st Joint Meeting of the British and Spanish Developmental Biology Societies

Alicia Hidalgo and Lola Martin-Bermudo

Int. J. Dev. Biol. (2009) 53: 443-446

\section{Mouse induced pluripotent stem cells}

Eamon Geoghegan and Lucy Byrnes

Int. J. Dev. Biol. (2008) 52: 1015-1022

A novel, neural potential of non-hematopoietic human umbilical cord blood stem cells Krystyna Domanska-Janik, Leonora Buzanska and Barbara Lukomska

Int. J. Dev. Biol. (2008) 52: 237-248

Pluripotency and differentiation in embryos and stem cells - Pavia, 17-18 January 2008

James A. Adjaye, Anne G. Byskov, Jose B. Cibelli, Ruggero De Maria, Stephen Minger, Maurilio Sampaolesi, Giuseppe Testa, Catherine Verfaillie, Magdalena Zernicka-Goetz, Hans Schöler, Michele Boiani, Nicola Crosetto and Carlo A. Redi

Int. J. Dev. Biol. (2008) 52: 801-809

$$
5 \text { yr ISI Impact Factor }(2009)=3.253
$$

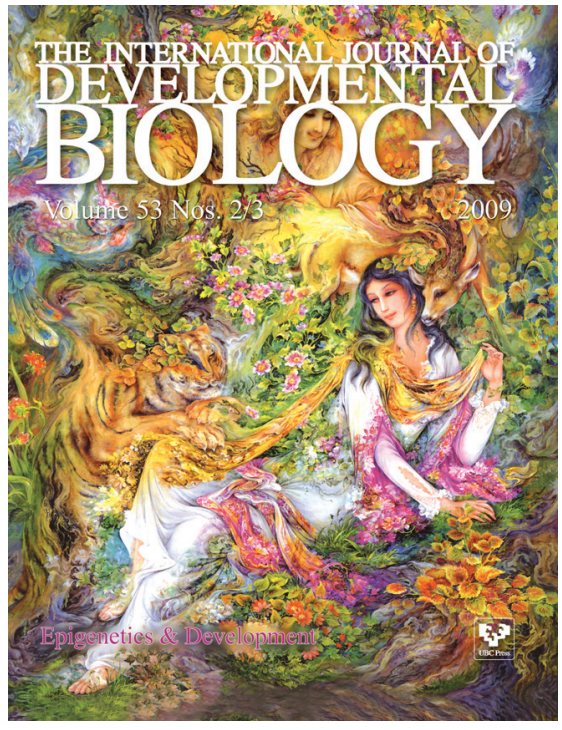

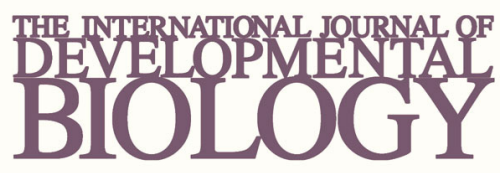

Volume 54 Nos. 6/7
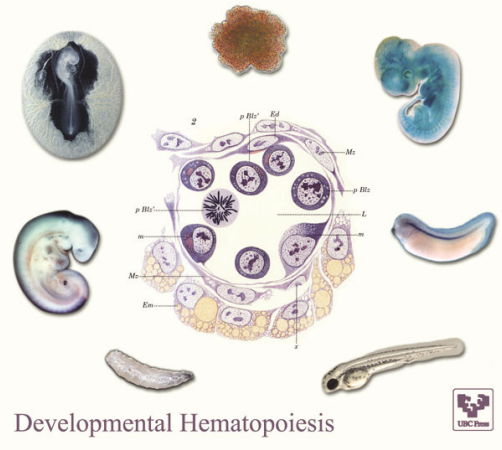

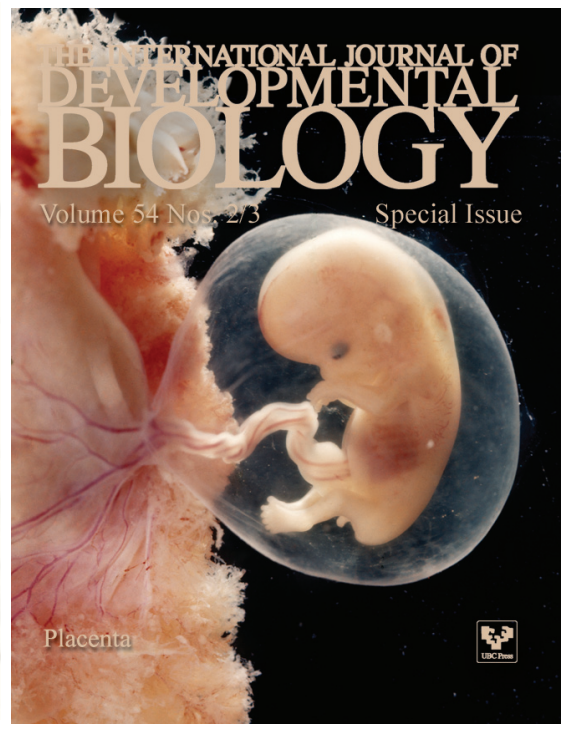

